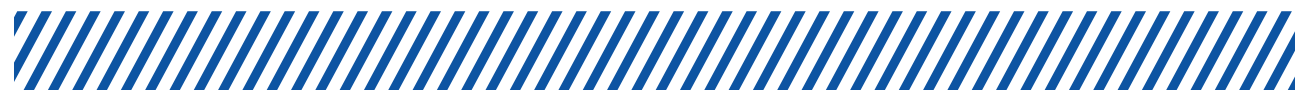

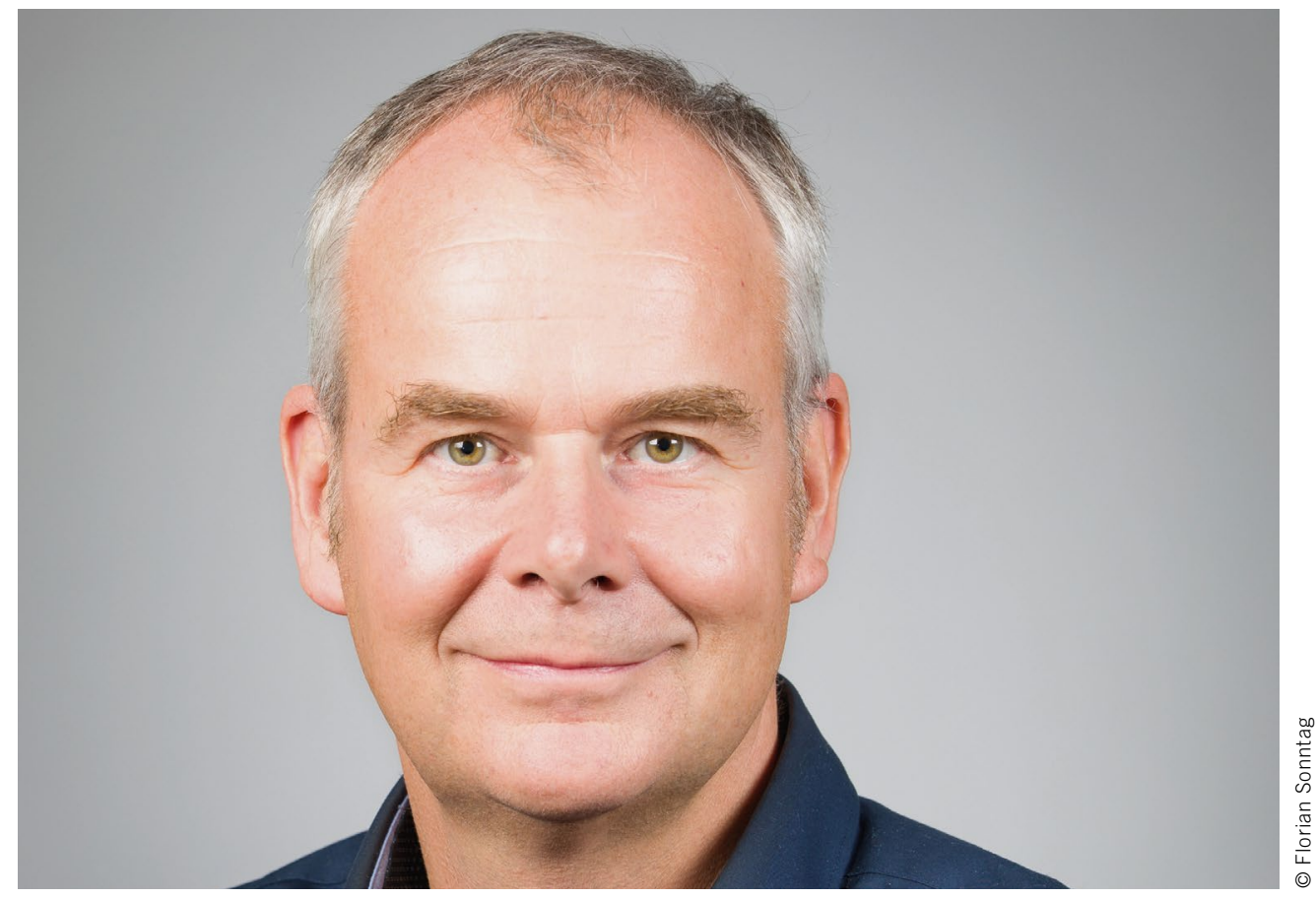

Dr. Karsten Wilbrand

Senior Principal Scientist at Shell

\section{We Need a Technology-neutral Approach}

The energy transition will involve replacing our existing fossilfuel-based system with the use of renewable energy. All sectors of the economy must be included in this process. The transport sector must not be regarded as a separate case. Although the focus is currently on electrification, we need a technology-neutral approach.

The trend for electrification applies not only to transport, but also to manufacturing industry and construction. For this reason, it seems not unrealistic to expect that electricity demand in Europe could double by 2050 . Even if we succeed in significantly increasing the generation of renewable electricity within Europe, the question remains of how to compensate for the fluctuation in renewable energy production by the means of the renewable generation of the baseload, improved grid integration and storage.

For the baseload, the new German government is putting the emphasis on the construction of modern gas-fired power stations that can be converted to operate on climate-neutral gases $\left(\mathrm{H}_{2}\right.$-ready). It believes that hydrogen will be of overriding importance for the energy transition. The aim is for Germany to become the leading market for hydrogen technology and increase its domestic electrolysis capacity to $10 \mathrm{GW}$ by 2030 . In December, the EU announced a package for decarbonizing the gas sector in which hydrogen also plays a key part.

Alongside the domestic production, hydrogen production is particularly suitable in areas where it will be highly cost-effective, sustainable and where large areas of land are readily available. Regions like North Africa, the Middle East, Patagonia and Australia are ideal candidates. Depending on transport distance and the intended use, the import-options include pipelines, liquid hydrogen in ships and synthetically produced hydrogen derivatives, such as methanol, ammonia and e-fuels.

If locally produced and imported hydrogen has to be reconverted into electricity in the future to enable us to meet our netzero emissions targets, this will put into perspective the discussion about the greater well-to-wheel efficiency of electric trucks (60\% in the Vecto cycle) compared with hydrogen trucks (24\%). An electric truck that is $50 \%$ charged with directly generated electricity and $50 \%$ with hydrogen reconverted to electricity has a well-to-wheel efficiency of just under $32 \%$. This does not take into account the fact that the same solar or wind power systems in suitable locations generate much more electricity than they would in Germany.

As the demand for green molecules for energy imports, storage and conversion back to electricity grows, the direct use of these molecules in the transport sector and a technology-neutral approach make significant sense. Depending on the application, battery electric or hydrogen-powered vehicles may be a better solution for road transport. In the case of applications with a very high power consumption, such as off-highway vehicles, shipping and aviation, the essential options for decarbonization from a medium- and long-term perspective, alongside biofuels, are hydrogen and e-fuels. 